nesium pyrophosphate as a qualitative test, for iron revealed its presence there. As sample No. 39 I contains 4.70 per cent. ferric oxide, this may account for the wider variation than in No. 389 between the gravimetric and volumetric results.

RALEIGH, ‥ C.

[CONTRIBUTIONS FROM THE CHEMICAI LABORATORY OF LAFAYETTE COILEGE.]

\title{
A METHOD FOR PREPARING NORMAL, SEMINORMAL, DECINORMAL, ETC., SULPHURIC ACID OF EXACT STRENGTH.
}

By Richard K. MEADE.

Received November I', Igoo.

THE

$\mathrm{HE}$ principal beauty of the normal system in volumetric analysis is the doing away with calculations. If then it is necessary to use a factor for converting to normal with a solution. much of the usefulness of the system is destroyed. Unfortunately with by far the larger number of reagents used in volumetric analysis it is only with the greatest care that such solutions can be made of normal strength. Of the commonly used acid and alkali solutions, only oxalic acid and sodium carbonate can be prepared of exact strength, without first having another standard solution against which to balance a preliminary and then the exact solution. Even then, the latter is frequently too wide of the normal value to be used without a factor. The writer has been for some time preparing normal, seminormal, and more particularly decinormal sulphuric acid of exact strength by the method given below. The solution as prepared by this method needs no checking, except as a safeguard against errors of manipulation on the part of the analyst.

If the electric current is passed through a solution of copper sulphate the salt is decomposed, copper separating upon the cathode and sulphuric acid at the anode. This latter remains in solution and is not decomposed by the current. Hart and Croasdale ${ }^{1}$ took advantage of this reaction to standardize alkali solutions. Their results were highly accurate. The writer has frequently made use of this method of standardizing alkali and has obtained results far more satisfactory than by any other method.

$1 J$. Anal. Chem., 4, 424. 
The method of preparing tenth-normal sulphuric acid by means of this reaction is as follows :

I 2.487 grams of pure crystallized copper sulphate are dissolved in about $750 \mathrm{cc}$. of distilled water in a lipped beaker capable of holding about a liter. Into this solution, after cooling, is introduced a cylinder of copper foil attached to the minus (-) wire of an electric circuit. This copper cylinder may be made from 0.015 inch copper foil. The foil is cut the required length (three times the diameter of the beaker - one-half inch) curled so that the ends lap, and holes punched through the two thicknesses of foil with a sharp tail. Wire is then run through the holes, fastening the two ends of the foil together. A platinum rod for an anode is next passed through a perforated watch-glass covering the beaker into the copper sulphate solution. A current of electricity of from one to one and a half amperes is now passed through the solution for about eight hours; or all night if the decomposition is begun in the afternoon. In the morning the watch-glass is removed and rinsed off together with the cylinder and the rod into the beaker. The solution is then transferred to a liter graduated flask ; any copper which may have dropped off the cylinder into the beaker is to be washed well by decantation, rinsing the beaker at the same time into the flask. The contents of the latter are then diluted to the mark.

The first few solutions made in this way were subjected to the following tests:

A portion of the solution was carefullynevaporated to small bulk and hydrogen sulphide passed into the liquid. No precipitate formed.

Another portion of the solution was evaporated to a few cubic centimeters, ammonia added, and the test-tube stood upon white paper. No blue color.

A third portion of the solution after evaporation was placed in a bright platinum dish, a drop of nitric acid added and a weak current passed through the solution. After one hour no copper stain appeared on the dish and the platinum was still bright.

The results given by the first solution made when checked by volumetric and gravimetric methods are given below :

An exactly tenth-normal solution of sodium carbonate, made by igniting pure bicarbonate and then dissolving 5.305 grams of 
the resulting sodium carbonate in a liter of water, was first used.
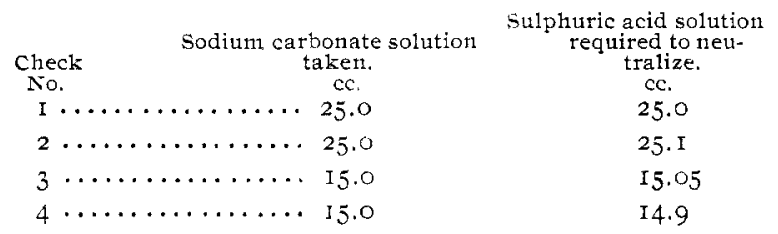

A tenth-normal sodium hydroxide solution was next used. This solution was made by dissolving freshly cut clean bright pieces of metallic sodium in water contained in a silver dish and covered with a large inverted funnel. It was preserved in a large bottle and drawn off for use by means of a siphon passing through the stopper of the bottle. All air entering the bottle passed through a soda-lime tube, capped when not in use. This sodium hydroxide solution had been checked against a fifth-normal hydrochloric acid solution which in its turn had been standardized by precipitation with silver nitrate. It was also checked by the copper sulphate and battery method of Hart and Croasdale. These checks established a factor $0.9950 \mathrm{I}$, for converting to tenth-normal.

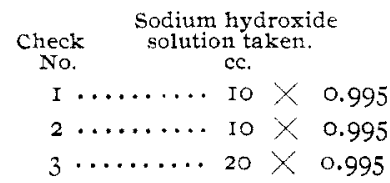

\begin{tabular}{|c|c|}
\hline $\begin{array}{l}\text { Equivalent rolume } \\
\text { of N/rosolution. } \\
\text { cc. }\end{array}$ & $\begin{array}{l}\text { Sulphuric acid } \\
\text { solution required. } \\
\text { cc. }\end{array}$ \\
\hline 9.95 & 10.0 \\
\hline 9.95 & 9.9 \\
\hline 19.9 & 19.9 \\
\hline
\end{tabular}

Finally $25 \mathrm{cc}$. of the sulphuric acid solution were acidified with a few drops of hydrochloric acid, heated to boiling, and an excess of a hot ro per cent. solution of barium chloride slowly added with constant stirring. After standing all night the precipitate was filtered off, ignited, and weighed.

I. Weight of barium sulphate. 2923 gram.

The molecular weight of barium sulphate is 233.46 ; hence, $25 \mathrm{cc}$. of a tenth-normal solution should give $\frac{233.46 \times 25}{2 \times 10 \times 1000}=0.29183$ gram barium sulphate. The solution is therefore a little under tenth-normal, or 2923:2918::x:1;x=1.002, which gives the factor for converting to tenth-normal.

2. Weight of barium sulphate $\ldots \ldots \ldots \ldots \ldots \ldots \ldots \ldots .29{ }^{8}$ gram.

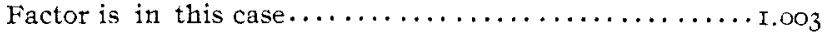

Average factor for the two determinations.............0025 
Of course results by precipitation with barium sulphate would be worthless, if the absence of copper had not been previously proved.

Since making this solution, the writer has repeatedly made normal and fifth-normal solutions by this method; it was not until recently though, that he had occasion to make a normal solution. In this case, 124.87 grams of copper sulphate were dissolved in $800 \mathrm{cc}$. of water and decomposed by a current of 2.5 amperes. The decomposition was complete in about twelve hours, though the current was allowed to run nearly eighteen. After making up to a liter, the strength of the solution was taken against the sodium carbonate solution mentioned above with the following results :

$\begin{array}{cc}\text { Sulphuric acid solution taken. } & \text { Sodium carbonate solution required. } \\ \text { cc. } & \text { cc. } \\ 5.0 & 50.1 \\ 5.0 & 50.2 \\ 5.0 & 49.8 \\ 5.0 & \frac{49.95}{50.025}\end{array}$

roo cc. of the normal solution were then diluted to rooo cc. in a graduated flask, and after mixing tested against the $\mathrm{N} / \mathrm{IO}$ sodium hydroxide solution mentioned above.

\begin{tabular}{|c|c|c|c|c|c|}
\hline $\begin{array}{c}\text { Sodium hydroxid, } \\
\text { taken. } \\
\text { cc. }\end{array}$ & obution & & \multicolumn{2}{|c|}{$\begin{array}{l}\text { Equivalent volume of } \\
\text { N/10 alkali solution. }\end{array}$} & $\begin{array}{l}\text { Sulphuric acid } \\
\text { required. } \\
\text { cc. }\end{array}$ \\
\hline IO.O & $x$ & 0.995 & $=$ & 9.95 & 10.1 \\
\hline 10.0 & $x$ & 0.995 & $=$ & 9.95 & 10.0 \\
\hline 10.0 & $x$ & 0.995 & $=$ & 9.95 & 10.0 \\
\hline 10.0 & $x$ & 0.999 & $=$ & 9.95 & 9.95 \\
\hline IO.O & $x$ & 0.995 & $=$ & 9.95 & 9.95 \\
\hline & & & & & 10.00 \\
\hline
\end{tabular}

All of the solutions made by this method have been checked against the sodium hydroxide solution, with the result that the strength of the sulphuric acid solution is always the desired one. 